\title{
Nanobodies as novel agents for disease diagnosis and therapy
}

\author{
This article was published in the following Dove Press journal: \\ International Journal of Nanomedicine \\ 31 October 2013 \\ Number of times this article has been viewed
}

\section{Christina G Siontorou \\ Department of Industrial Management and Technology, University of Piraeus, Piraeus, Greece}

\begin{abstract}
The discovery of naturally occurring, heavy-chain only antibodies in Camelidae, and their further development into small recombinant nanobodies, presents attractive alternatives in drug delivery and imaging. Easily expressed in microorganisms and amenable to engineering, nanobody derivatives are soluble, stable, versatile, and have unique refolding capacities, reduced aggregation tendencies, and high-target binding capabilities. This review outlines the current state of the art in nanobodies, focusing on their structural features and properties, production, technology, and the potential for modulating immune functions and for targeting tumors, toxins, and microbes.
\end{abstract}

Keywords: heavy chain antibodies, nanobodies, antibody expression, molecular display, formatting

\section{Introduction}

Antibodies (Abs) represent essential research tools and a well-established class of clinical diagnostics. The advent of molecular engineering and phage display technology facilitated the extension of $\mathrm{Ab}$ applications to molecular imaging and therapeutics for several major diseases, including autoimmune, cardiovascular, and infectious diseases, cancer, as well as inflammation. Abs can be used as unarmed therapeutic agents that inhibit targets involved in disease progression, or by causing the cytotoxic death of target cells, which are mediated by modulators of the immune response; alternatively, Abs can act as carriers of cytocidal and imaging agents. Their applicability presupposes careful engineering of their biochemistry to fit their intended use, aiming at improving effector functions, producing $\mathrm{Ab}$-drug conjugates, and downsizing. The former approach, involving technically demanding and costly procedures such as grafting and directed mutagenesis, may not always be successful, as judged in numerous clinical trials. ${ }^{1-3}$ The conjugation of proteins, toxins, or radionuclides converts Abs to drug delivery systems or inactive prodrugs that can selectively interact with targets. This strategy requires two or three separate components (the Ab, linker, and conjugate) and various treatment steps that, in theory, could produce homogeneous, specific, stable, soluble conjugates, with high affinity and no aggregation- and proteolysis-susceptible regions. ${ }^{4}$ In practice, the high immunogenic potential of the linkers and the broad diffusion of the conjugate result in marginal therapeutic indices; ${ }^{5}$ efficacy is expected to improve by the use of nanodelivery technology, once the pending issues in production are resolved and the in vivo pharmacokinetics are calculated. ${ }^{6}$ The large molecular size of Abs $(\sim 150 \mathrm{kDa})$ results in long serum half-lives, which causes dose-limiting myelotoxicity, ${ }^{6}$ limited target penetration efficacy, ${ }^{5}$ and a high background in imaging. ${ }^{7}$ The
Correspondence: Christina G Siontorou Department of Industrial Management and Technology, University of Piraeus, 80 Karaoli and Dimitriou Street, 18534 Piraeus, Greece Tel +302104142368 Fax +302104142392

Email csiontor@unipi.gr 
pharmacokinetic requirements depend upon the intended use: in therapy, the prolonged in vivo retention enhances bioavailability and simplifies drug administration; ${ }^{3}$ in drug delivery, rapid elimination is preferable to avoid tissue toxicity. ${ }^{6}$

Another avenue to explore comes with $\mathrm{Ab}$ fragmentation. Downsizing - with the use of enzymes or solvents, and multistep processes - has progressed from antigen-binding fragments (Fabs; $\sim 57 \mathrm{kDa}$ ) to single-chain variable fragments (scFvs; $\sim 27 \mathrm{kDa}$ ). Compared to the intact $\mathrm{Ab}$ structure, the Fab shows an increased capacity to penetrate the dense tissue of solid tumors and scFv seems to be even more effective. Following the research hypothesis of enhancing potency with reducing size, third-generation fragment technology delivers single-cell and miniaturized Abs $(\sim 11-15 \mathrm{kDa})$. The size advantage is, however, counterbalanced by lower affinities, ${ }^{8}$ difficulties in mass production, ${ }^{9}$ a very short serum half-life that disfavors the slower uptake kinetics, ${ }^{10}$ and a tendency to aggregate. ${ }^{11}$

The discovery in the early 1990s that Camelidae (bactrian camels, dromedaries, vicugnas, and llamas) produce fully functional $\mathrm{Ab}$ structures that retain only the most essential antigen-binding regions ${ }^{12}$ demonstrated the superiority of nature in downsizing, but it also verified the research hypothesis. These structures lack the light chains of conventional Abs and are known as heavy-chain Abs (HCAbs). Although similar structures have also been identified in elasmobranch cartilaginous fish (sharks, rays, and skates), ${ }^{13,14}$ most research has been performed on camelids because of their ease of handling and immunization. ${ }^{15}$ Derived from evolutionary processes, HCAbs possess certain features that facilitate their further splitting to stable, soluble, and easily manipulated single-domain ( $\mathrm{sdAbs}$ ) formats, used to deliver a variety of derivatives. Owing to a highly lucrative market, academic spin-offs were fast to capitalize on the new moieties, with a few products currently in clinical trials, from the Belgian Ablynx (www.ablynx.com) on the camelid source and the US GenWay Biotech (www.genwaybio.com) on the shark source. During the uprising nanotechnology era, Ablynx dubbed these moieties Nanobodies ${ }^{\circledR}$ in 2003 to promote their nanosize $(2.5 \mathrm{~nm}$ in diameter and $4 \mathrm{~nm}$ in height). ${ }^{15}$ In the meantime, the efforts to reengineer $\mathrm{Ab}$ fragments to nanobody $(\mathrm{Nb})$ size and function continued, with GlaxoSmithKline (Brentford, UK) acquiring Domantis to get a hold of its human-derived sdAbs.

Following the proof-of-concept, HCAbs have started to intrigue the scientific community as new Ab-based tools. In order to examine the dynamics of the research paths (ie, to determine technology boundaries and growth, and to decide on the incorporation of relevant terms and concepts that have been used interchangeably for nature-derived sdAbs, including $\mathrm{Nb}$, sdAb, $\mathrm{HCAb}$, single-chain $\mathrm{Ab}$, and so on), the use of ontological data acquisition and mining tools has proven beneficial. ${ }^{16-18}$ The number of publications on Camelidae $\mathrm{HCAbs}$ and $\mathrm{Nbs}$ has risen dramatically since 2008 , totaling up to 1,210 original articles in the Web of Science ${ }^{\circledR}$ database (Thomson Reuters, Philadelphia, PA, USA) published within 9 years (2004-2012) from 300 universities in 67 countries to cover the areas of molecular biology, immunology, hematology, and experimental medicine (Figure 1). The 10-year exploration phase, predominantly oriented to the elucidation of $\mathrm{Nb}$ structure and properties, ${ }^{13-15,19-24}$ was quickly followed by a rapidly increasing exploitation phase (Figure 2). ${ }^{25-58}$ This fast transition was enabled by the existing technological frame that offered an established research environment in terms of accumulated knowledge, capital outlays, infrastructure, and available skills. The scientific network, developed mostly by central European and US clusters, is field-specific and concrete, pushing and pulling players into finite sets of positions according to the needs for knowledge absorption. The number of possibilities and prospects with the technology at hand are numerous. This review outlines the current state of the art in $\mathrm{Nbs}$, focusing on $\mathrm{Nb}$ structural features and properties, as well as its production and technology potential that also bears prospects for exploitation in other biotechnological fields.

\section{Conventional antibodies and single-domain antibodies Conventional antibodies and antibody fragments}

Immunoglobulin $(\mathrm{Ig}) \mathrm{G}$, one of the five isotypes found in humans and the only one that crosses the placenta, provides the majority of Ab-based immunity and comes in four forms: $\mathrm{IgG1}, \mathrm{IgG} 2, \mathrm{IgG} 3$, and IgG4. IgG1 is primarily employed in therapeutics, providing a clear advantage in enhancing effector functions and offering a longer serum half-life ( $\sim 21$ days). ${ }^{1-3}$ It has a basic heterotetrameric structure and is composed of two identical heavy $(\mathrm{H})$ chains covalently linked by disulfide bonding, and two identical light (L) chains (Figure 3A). The $\mathrm{H}$-chain folds into four domains: one variable $(\mathrm{VH})$ and three constant $(\mathrm{CH} 1, \mathrm{CH} 2$, and $\mathrm{CH} 3)$; whereas the $\mathrm{L}$ chain consists of a variable (VL) and a constant (CL) domain that interact noncovalently with the $\mathrm{VH}$ and $\mathrm{CH} 1$ domains, respectively. These covalent and noncovalent associations result in the formation of three independent regions: two Fabs and one 


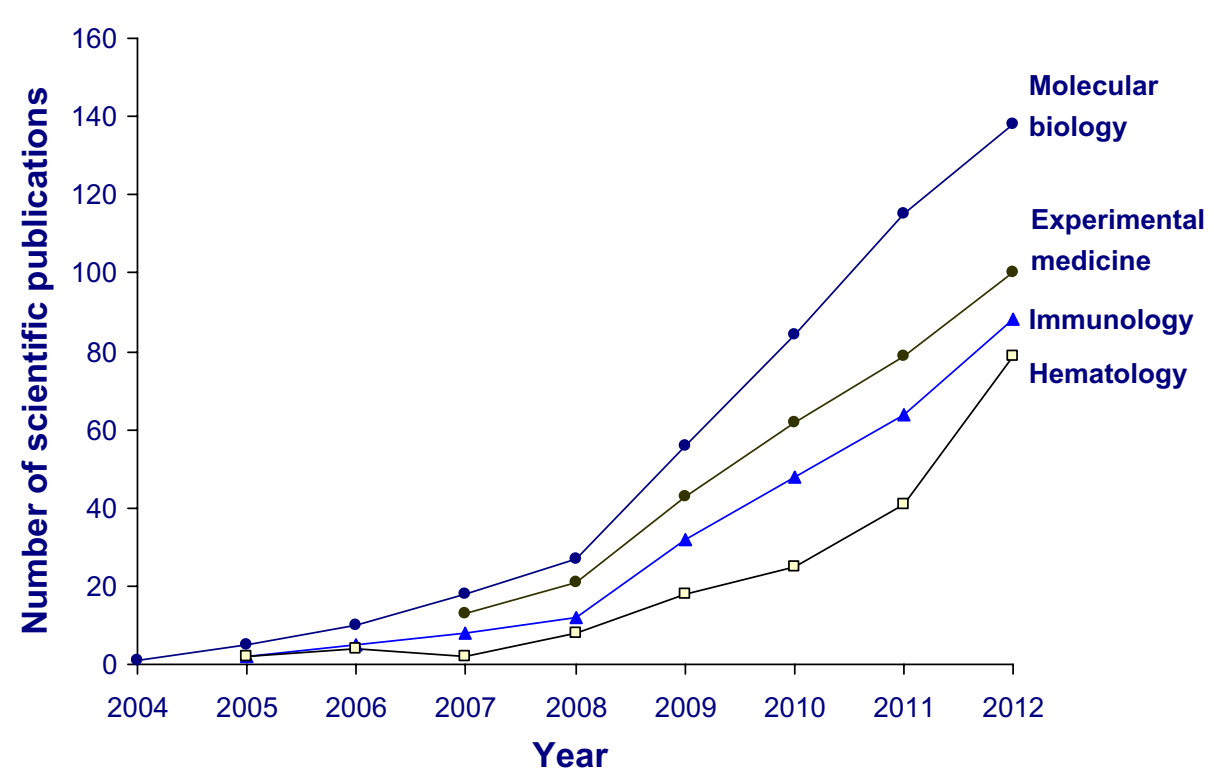

Figure I Nb research trends (2004-20I2) placing emphasis on original works on Camelidae HCAbs retrieved from the Web of Science ${ }^{\circledR}$ database (Thomson Reuters, Philadelphia, PA, USA).

Notes: The clustering into scientific fields is based on the science overlay mapping approach, ${ }^{16-18}$ which uses the subject categories that the Web of Science ${ }^{\circledR}$ assigns to journals: for a set of publications indexed by the Web of Science ${ }^{\circledR}$; Nbs are located via the journals in which they appear. The assignment of each paper into a scientific field is determined by the classification of (i) the cited references in the paper and (ii) the citations that the paper received.

Abbreviations: Nb, nanobody; HCAbs, heavy-chain antibodies.

crystallizable fragment $(\mathrm{Fc})$, connected through a flexible linker at the hinge region. The Fab regions are of identical structure, usually flat or concave, wherein each expresses a specific antigen-binding site. The Fc region determines in vivo retention and expresses the interaction sites for ligands that can induce effector functions, which are mostly governed by the glycoform of an oligosaccharide covalently attached to $\mathrm{CH} 2$ at the asparagine 297 position. ${ }^{3}$ The paired $\mathrm{N}$-terminal $\mathrm{VH}-\mathrm{VL}$ domains constitute the paratope or variable fragment (Fv).

During the production of conventional Abs from plasma B-cells, somatic recombination yields a mature message that is transported to the cytoplasm and is translated in the endoplasmic reticulum into an $\mathrm{H}$-chain that is bound to chaperon proteins; these proteins, along with the $\mathrm{CH} 1$, facilitate its placement over the contact sites of the L-chain domains. ${ }^{59}$ The CH1 spaces, also, the two antigen-binding sites by $80-160$ $\AA$ that optimizes recognition and cross-linking of repeated epitopes. Well conserved hydrophobic amino acids, at positions 37 (valine), 44 (glycine), 45 (leucine), and 47 (tryptophane) in the $\mathrm{VH}$ region, form the major interaction site of the chaperon proteins. The folded V-domain comprises nine $\beta$-strands connected by canonical loops and by a conserved disulfide bond between cysteine 23 and cysteine 94, which are packed against a conserved tryptophan. ${ }^{59}$ In this way, hypervariable regions are formed (called the complementarity determining regions [CDRs]), and there are three each on the $\mathrm{VL}$ and the VH chains that determine specificity, diversity, and affinity; the remainder of the VH and VL domains are called framework regions and support the loops. ${ }^{8}$ In human and mouse VHs, the number of loop architecture combinations is limited.

The exposed hinge region is extended in the structure due to its high proline content and is therefore vulnerable to proteolysis; papain can split the Ab into Fab and Fc fragments (Figure 3B). Other proteolysis-susceptible sites do exist, and the use of various enzymes or solvents is used to produce smaller fragments. In recombinant approaches, the VL and VH of the Fv fragment are commonly attached via a flexible polypeptide linker to produce an $\mathrm{scFv}$ with a monovalent nature (Figure 3C) that decreases avidity and gives a very short serum half-life that limits applicability. ${ }^{11}$ Multimerization can improve retention and affinity, whereas the insertion of disulfide bonding in the framework produces a more stable fragment (called disulfide stabilized Fv). The combination of two different scFvs results in bispecific constructs. ${ }^{2}$ Further engineering can provide sdAbs that have either the VH or the VL domain; each sdAb contains three of the six naturally occurring CDRs from the parental $\mathrm{Ab}$. The initial attempts with mouse VH or VL scaffolds were soon repeated with human VH scaffolds to generate sdAbs. ${ }^{60,61}$ These mini-forms exhibit satisfactory 


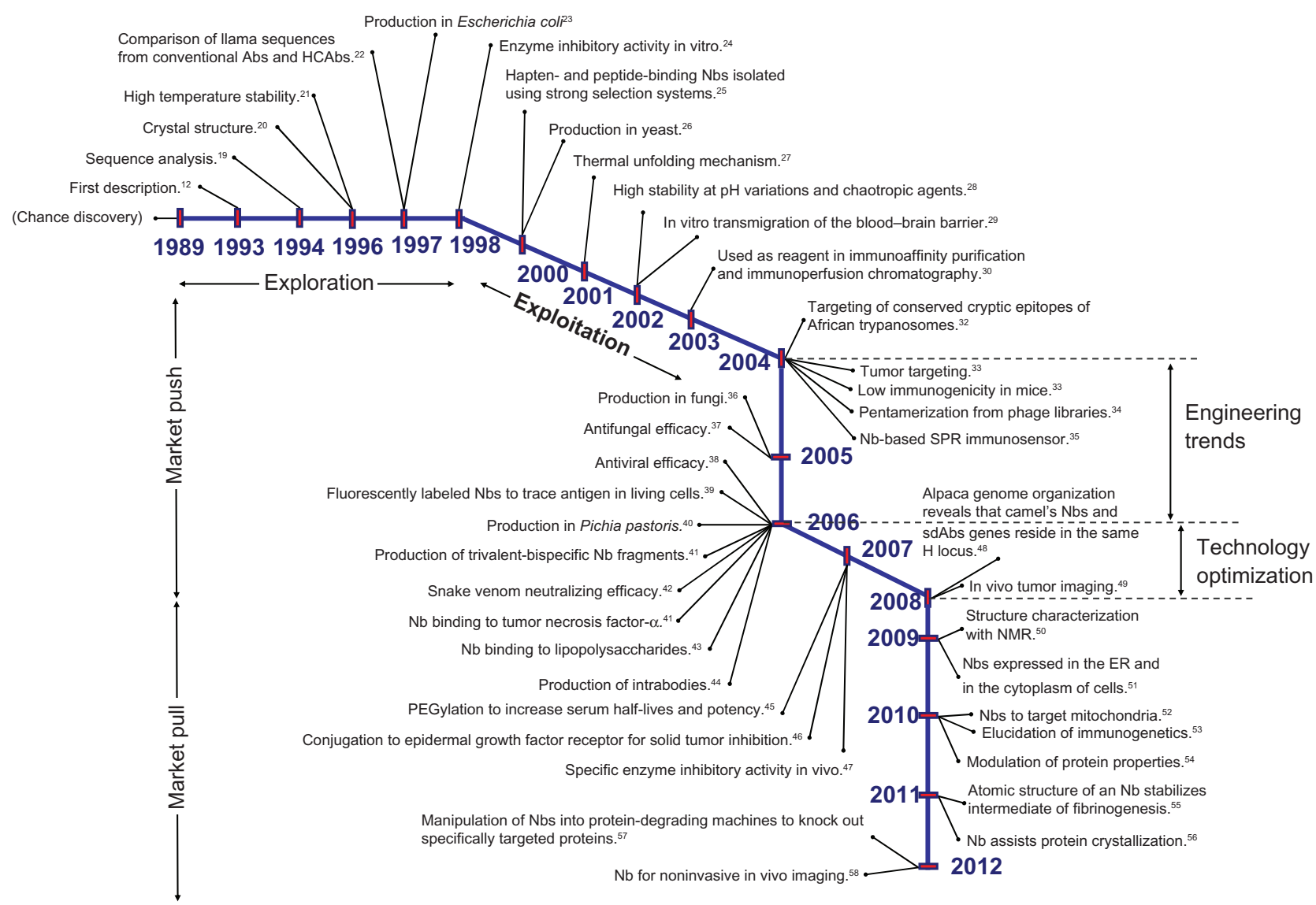

Figure 2 The scientific roadmap of university-derived advancements in Camelidae HCAbs.

Notes: Since their discovery in 1989, research groups moved fast to integrate Nbs in the well-established scientific frame of Ab-based biomedical tools. Although a clear distinction between the exploration and the exploitation phase cannot be made, and since even the latest papers are, to some extent, concerned with elucidation of mechanisms and physical chemistry, while the applicability domain was clearly defined in the introductory paper of 1993, a turning point can be found around 1998. As the spinoff, Ablynx, was moving to patenting and clinical testing at the early 2000 s, the academic research groups were feeding a knowledge push mechanism until 2008 , offering relations between properties and potential (until 2004), engineering trends (until 2006) and technology optimizations (until 2008). More researchers were attracted to join and extend the scientific network. Thereafter, the transition to a market pull mechanism becomes apparent, justifying Nbs as viable alternatives to Ab fragments, while a clear therapeutic advantage remains to be clinically proven. Superscripted numbers refer to published papers that document either a Nb asset or a stated intended use.

Abbreviations: Ab, antibody; HC, heavy chain; Nb, nanobody; SPR, surface plasmon resonance; PEGylation, polyethylene glycol treatment; H, heavy; sd, single-domain; NMR, nuclear magnetic resonance; ER, endoplasmic reticulum.

stability in vivo and are more suitable for complex manifold constructs.

\section{Naturally occurring heavy chain antibodies}

In addition to conventional $\operatorname{IgG} 1$, a portion of $\operatorname{IgG} 2$ and IgG3 of Camelidae (Tylopoda suborder) are HCAbs lacking the L-chain polypeptide and the first constant domain (CH1); their antigen-binding site is located in a $\sim 15 \mathrm{kDa}$ single variable domain called VHH (Figure 3D). The alignment of the VHH amino acid sequences indicated that the structural organization of the framework and hypervariable regions is similar to $\mathrm{VH}$, with a few significant differences in the framework-2 region and in the CDRs. ${ }^{22}$ The percentage of HCAbs varies greatly among taxa due to a variation in mutation rates. ${ }^{19-22}$ The exact mechanism remains obscure; these forms are derived from conventional Abs within the Tylopoda lineage as the outcome of adaptive changes. ${ }^{61}$

Camelids carry a nucleotide $\mathrm{G}$ to a point mutation that disrupts the consensus-splicing site at the $5^{\prime}$ end of the intron between the CH1-hinge exons and provokes the elimination of the $\mathrm{CH} 1$ region from the messenger ribonucleic acid by splicing. ${ }^{62}$ The hydrophobic amino acids found in the conventional $\mathrm{VH}$ region at positions $37,44,45$, and 47 are replaced by hydrophilic phenylalanine, glutamic acid, arginine, and glycine, respectively, thus depriving the H-chains of their binding sites for the chaperon proteins. ${ }^{59}$ The truncated and more soluble H-chains cannot be retained by the chaperon system and escape from the endoplasmic reticulum. The presence of more polar and charged residues enhances solubility and reduces aggregation. ${ }^{12,19,22}$ Solubility is further supported 


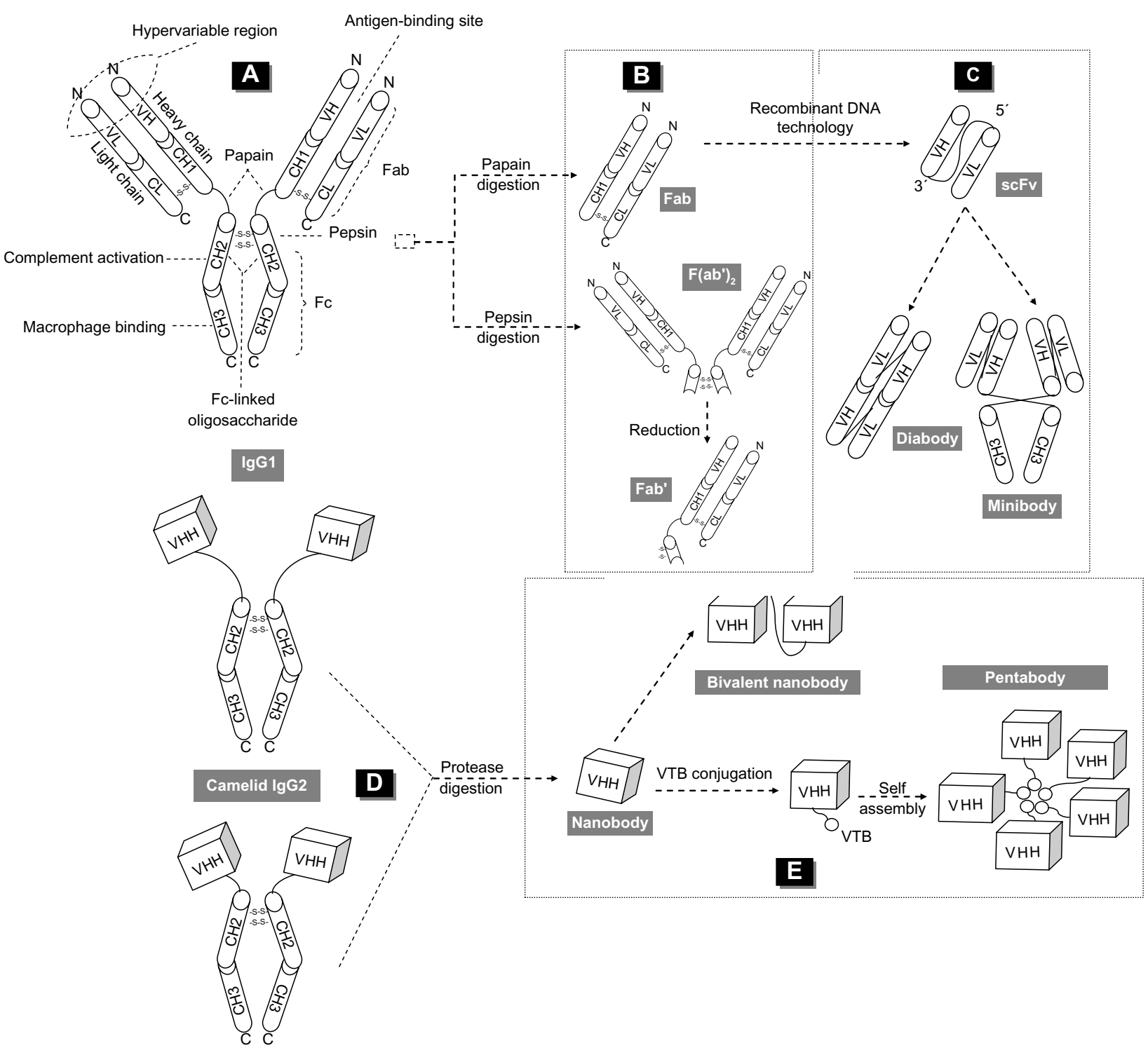

Camelid $\lg \mathrm{G} 3$

Figure 3 Schematic representations of intact IgGI, fragmentation products, engineered fragments and minibodies, IgG2 and IgG3, and the VHH domain or nanobody.

Notes: Schematic representations of $(\mathbf{A})$ intact $\operatorname{lgG}$ I; (B) fragmentation products; (C) engineered fragments like diabodies, formed by interlinking two scFv molecules via a short peptide, and minibodies with a diabody structure equipped with an additional CH3 domain; (D) IgG2 and lgG3 HCAbs in the sera of camelids; and (E) the VHH domain or nanobody, which can be easily engineered to bispecific formats or conjugated to the VTB, which self assembles into a homopentamer.

Abbreviations: $\mathrm{VH}$, variable heavy chain; $\mathrm{CH}$, constant heavy chain; $\mathrm{VL}$, variable light chain; $\mathrm{CL}$, constant light chain; Fab, antigen-binding fragment; $\mathrm{Fc}$, crystallizable fragment; scFV, single-chain variable fragment; VHH, single-variable domain; Ig, immunoglobulin; VTB, B-subunit of Escherichia coli verotoxin; HCAbs, heavy-chain antibodies.

by a mutation at position 11 (serine instead of leucine) which is, however, less well-conserved in llamas. ${ }^{22}$ The spacing of the antigen-binding sites, guided by $\mathrm{CH} 1$ in conventional $\mathrm{VHs},{ }^{59}$ is sufficiently sustained in VHHs by the rod-like long hinge. The hinges can be cleaved using proteases to deliver the isolated VHH domain or the $\mathrm{Nb}$ (Figure 3E). In effect, the technology developed for the scFv fragments can also be used for $\mathrm{Nb}$ isolation and formatting, which is more easily multimerized to generate constructs with a variety of avidity effects or bispecificity.
Solubility and the antigen-binding capability of $\mathrm{Nbs}$ are also favored by significant differences from VHs in the hypervariable regions. Their scaffolds consist of two $\alpha$-sheeted structures that are similar to VHs. CDR1 is extended toward its $\mathrm{N}$-terminal by four more amino acids that are, most probably, involved in antigen recognition. ${ }^{22}$ The CDR2 loop structures differ nearly always from the canonical loop structures that are defined for VHs, although the key residues are preserved. ${ }^{28}$ The CDR3 loop is longer and folds back to cover the former VL interface; ${ }^{19,20}$ this suggests an 
increase in the actual antigen-binding surface area, possibly compensating for the absence of antigen interactions provided by the VL partner in an Fv, and contributes to solubility. ${ }^{19}$ The broader structural repertoire of loops might contribute to $\mathrm{Nbs}$ recognizing alternative epitopes on an antigen. ${ }^{32}$ It was suggested recently that variable genes displaying a high degree of homology to the human VH4 family add to $\mathrm{HCAb}$ antigen-binding diversity. ${ }^{63}$

The CDR3 elongations can extend into cavities on antigens, such as the active site crevice of enzymes; indeed, the immune system of camelids seems to possess an inherent tendency to form enzyme-blocking HCAbs. ${ }^{50}$ However, an extended loop implies larger flexibility, and this is expected to be entropically counterproductive for binding. This is avoided in camelids by stabilizing their CDR3 loops with an additional disulfide bond toward either the CDR1 loop in camels or the CDR2 in llamas. ${ }^{63}$ These bridges may also maximize the topology on the binding surface and guide the orientation of the CDR3 towards the antigen.

\section{Production of nanobodies $\mathrm{HCAb}$ repertoire cloning}

Cloning HCAbs from an immunized camelid is a simple, inexpensive, and straightforward process that requires 2-6 boosters within 3-6 months. The titer and diversity of the response in HCAbs seem to depend on the actual antigen: small hydrophobic haptens tend to give weaker responses than larger antigens. Early attempts to produce polyclonal $\mathrm{Nbs}$ involved protease digestion and protein $\mathrm{A}$ or protein $\mathrm{G}$ purification. Variations in the IgG subisotype titers among taxa and problems in chromatographic separation gave moderate to low yields. ${ }^{12,19,21,62}$ The use of differential adsorption in affinity chromatography improved this process, which is now used in low-volume production. ${ }^{64,65}$ Most $\mathrm{Nb}$ market applications depend on large-volume, cost-effective, and reliable production; thus, cloning the repertoire of Fabs from an immunized camelid into a phage or ribosome display vector, as well as the selection of antigen-specific clones by panning, seem to be the methods of choice.

The peripheral blood lymphocytes collected from the immunized animal are used for messenger ribonucleic acid isolation and complementary deoxyribonucleic acid (DNA) synthesis. The gene fragments encoding the VHHs are specifically amplified by polymerase chain reaction (PCR). Although it is possible to amplify the entire VHH repertoire present within the blood sample, as all the VHHs belong to the same family and are encoded by an exon with homologous border sequences, the sticky behavior of VHs makes antigen-specific selection difficult. ${ }^{23}$ Hinge-specific or constant region-specific primers can be used to eliminate the $\mathrm{VH}$ gene fragments. ${ }^{62}$ The yield of the former is variable and often low, ${ }^{21,26}$ while the latter results in two types of primary PCR products that differ in length: the longest derives from the conventional $\mathrm{Ab}$, and the shorter from the $\mathrm{HCAb}$. The VH sequences are easily eliminated by agarose gel electrophoresis. ${ }^{62}$ A secondary PCR with nested primers annealing at either end of the $\mathrm{VHH}$ produces more material and allows the inclusion of restriction enzyme sites for cloning in an expression vector. ${ }^{15,19}$ The in vivo maturation of the HCAbs gives relatively small and manageable libraries $\left(10^{6}-10^{7}\right.$ individual clones). ${ }^{34,60,65}$ Selection can be performed by direct colony screening or by panning with standard enzyme-linked immunosorbent assay. Panning is generally preferred, as it allows for the selection of binders with the highest affinities, as well as those that express better in bacteria over several sequential rounds. ${ }^{62,64} \mathrm{Nb}$ libraries generated from immunized animals retain full functional diversity. This presents a significant advantage of Nbs over conventional $\mathrm{Ab}$ recombinants, which require reshuffling of VL and VH domains during library construction that may yield reduced diversities.

Despite the easy retrieval of high-affinity $\mathrm{Nbs}$ in a short period of time, immunization might not be possible due to low immunogenicity or high toxicity, or due to the limited availability of the antigen. ${ }^{66,67}$ Nonimmune libraries could be used to select binders with good specificity but $\mu \mathrm{M}$ affinity. ${ }^{60}$ Such libraries need to be larger $\left(\sim 10^{9}\right.$ individual clones $),{ }^{15}$ whereas subsequent and time-consuming in vitro affinity maturation steps should be included. ${ }^{66,67}$

\section{$\mathrm{Nb}$ expression systems}

$\mathrm{Nbs}$ are easily expressed in microbial systems (Escherichia coli, yeasts, fungi) from a single gene requiring no posttranslational modifications, thus allowing for shorter development times under well-established and scalable fermentation conditions. ${ }^{23,62}$ Unlike conventional VHs, the hydrophilic mutations in the VHHs contribute to high-expression yields. ${ }^{26,45}$ The high refolding capability of VHHs has also been correlated with a high production level in E. coli (up to $10 \mathrm{mg} / \mathrm{L}$ ). ${ }^{23,40,61}$ Despite the satisfactory yields, expression levels of different clones can vary by a factor of $100 ;{ }^{26,45}$ the use of random molecular evolution using DNA shuffling may improve production. ${ }^{21}$

Attempts to use filamentous fungi resulted in limited proteolytic degradation of the secreted product, ${ }^{36}$ possibly due to the 
high levels of the fungi proteases. ${ }^{68}$ High yields can be obtained from yeast systems, like Saccharomyces cerevisiae. ${ }^{38,69}$ These systems may also coproduce N-glycosylated Nbs with yeastspecific high-mannose oligosaccharides ${ }^{26,45}$ which, due to their binding to specific mannose receptors on reticuloendothelial cells, ${ }^{70}$ could increase the in vivo immunogenicity and decrease the serum half-life of the moiety.

The single-domain nature facilitates molecular manipulation and formatting. Genetic fusions of two or three Nbs can produce bivalent or high-avidity multimers. ${ }^{28,41,45,46}$ Alternatively, the $\mathrm{Nb}$ can be cloned to a protein with a natural tendency to dimerize or multimerize, such as a leucine zipper motif or the pentamerizing motif of verotoxin (Figure $3 \mathrm{E}$ ). ${ }^{34}$ Although multimerization is less efficient than the production of the monovalent format, yields are generally more satisfactory than those of conventional Ab constructs, as more flexible linkers can be used, and domain mispairing is avoided. Nonetheless, the steric hindrance by the N-terminal VHH may compromise antigen binding by the $\mathrm{C}$-terminal. ${ }^{32}$

Effector functions can be only recruited indirectly and to a lesser degree than in conventional Abs. Options include either the use of bispecific $\mathrm{Ab}$ fragments binding to host $\mathrm{IgG}$, or of $\mathrm{Nb}$ fusion to host Fc domains. ${ }^{62}$ Functionalization usually requires the correct glycosylation of the $\mathrm{CH} 2$ domain, which is feasible only in higher eukaryotic cells. ${ }^{11}$ The use of the glycoengineered Pichia pastoris expression system can generate specific $\mathrm{Nb}$-glycoforms by surface display; the library of $\mathrm{Nb}$-coding sequences can be fused to the $\mathrm{C}$-terminal part of the $S$. cerevisiae $\alpha$-agglutinin gene ( $S A G 1)$ and expressed in P. pastoris. A high-efficiency transformation protocol has been reported that yielded a library of $5 \times 10^{7}$ clones, $\sim 80 \%$ of which strongly expressed the $\mathrm{Nb}$ fusion. ${ }^{71}$

\section{Therapeutic applications, safety, and tolerability}

The unique physicochemical and pharmacokinetic properties of $\mathrm{Nbs}$ match the requirements of many biomedical applications (Table 1) and offer several advantages over conventional Ab technology for immunotherapy, drug delivery, and diagnostics. The affinities of Nbs are comparable or superior to those of conventional $\mathrm{Ab}$ derivatives, ${ }^{15} \mathrm{Nbs}$ with $100 \mathrm{pM}$ affinity constants have been isolated..$^{35,50}$ The fast retrieval of high-affinity and target-specific Nbs provides a rich pool for intracellular signaling molecules, protein-protein interactions, and cancer biomarkers. Nbs can be fused with fluorescent proteins to produce chromobodies that may be utilized in single-molecule localization with super-resolution imaging techniques. ${ }^{39,57}$ Since they can be engineered to induce conformation changes or to discriminate between conformational variants, Nbs may prove to be a beneficial research tool for monitoring protein expression, translocation, and subcellular localization. ${ }^{51,54}$

$\mathrm{Nbs}$ are easily concentrated by ultrafiltration to $1-10 \mathrm{mg} / \mathrm{mL}$ in standard phosphate or Tris buffers for usage as a stock solution. ${ }^{15}$ Their shelf-life ranges between a few months at $4{ }^{\circ} \mathrm{C}$ to several months at $-20^{\circ} \mathrm{C}$ while maintaining full antigen-binding capacity; they retain $>80 \%$ of their binding activity after 1 week of incubation at $37^{\circ} \mathrm{C}$ and may remain functional after several weeks of incubation. ${ }^{23}$ The hydrophilic content and the CDR3 loop folding confer thermal tolerance, aggregation resistance, and conformational stability at extreme $\mathrm{pH}$ values and in the presence of chaotropic agents. ${ }^{28}$ Melting points are in the range of $67^{\circ} \mathrm{C}-78^{\circ} \mathrm{C},{ }^{50}$ with thermal unfolding often shown to be fully reversible; functional activity can be retained up to $90^{\circ} \mathrm{C} .{ }^{21}$ The ruggedness of $\mathrm{Nbs}$ is suitable for harsh-condition engineering, ${ }^{27,44}$ tumor targeting, ${ }^{15}$ and surfactant-based pharmaceutical formulations. ${ }^{37}$

Further refolding of VHHs only requires domain refolding, thus avoiding the association of $\mathrm{VH}$ and $\mathrm{VL}$ domains in conventional Abs. The introduction of cysteines at position 54 and 78 result in an extra stabilizing disulfide bond at the buried hydrophobic region. ${ }^{72,73}$ These proteolysis-resistant platforms can be utilized in oral immunotherapy. Trypsinresistant $\mathrm{Nbs}$ for oral delivery have been obtained after random mutagenesis through DNA shuffling and selection for trypsin-resistant variants. ${ }^{74} \mathrm{Nbs}$ that successfully prevented diarrhea caused by rotavirus in a mouse model were similarly selected for resistance against the acidic environment of the stomach. ${ }^{38}$ Proteolysis can also be prevented by expressing $\mathrm{Nbs}$ in gut commensal bacteria, such as Lactobacilli, that have been used to produce rotavirus-neutralizing Nbs. ${ }^{75}$

Nbs can be genetically customized to target enzymes, transmembrane proteins, or molecular interactions. Their ability to recognize recessed antigenic sites has been attributed to their smaller size and the ability of the extended CDR3 loop to quickly penetrate into such epitopes. ${ }^{20,50}$ When fused to a truncated apolipoprotein L-1 enzyme, Nbs can specifically recognize the conserved carbohydrate of the surface glycoprotein found in Trypanosoma rhodesiensis, a sleeping sickness parasite variant. ${ }^{32,64} \mathrm{Nbs}$ genetically fused to $\beta$-lactamase can target the carcinoembryonic antigen; ${ }^{33}$ the enzyme then converts an injected nontoxic prodrug into a high-concentration toxic drug in the vicinity of the targeted tumor cells. Satisfactory results in mice are very promising for the Ab-based enzyme prodrug therapy approach. 
Table I Correlation between the biophysical properties of nanobodies with their areas of application

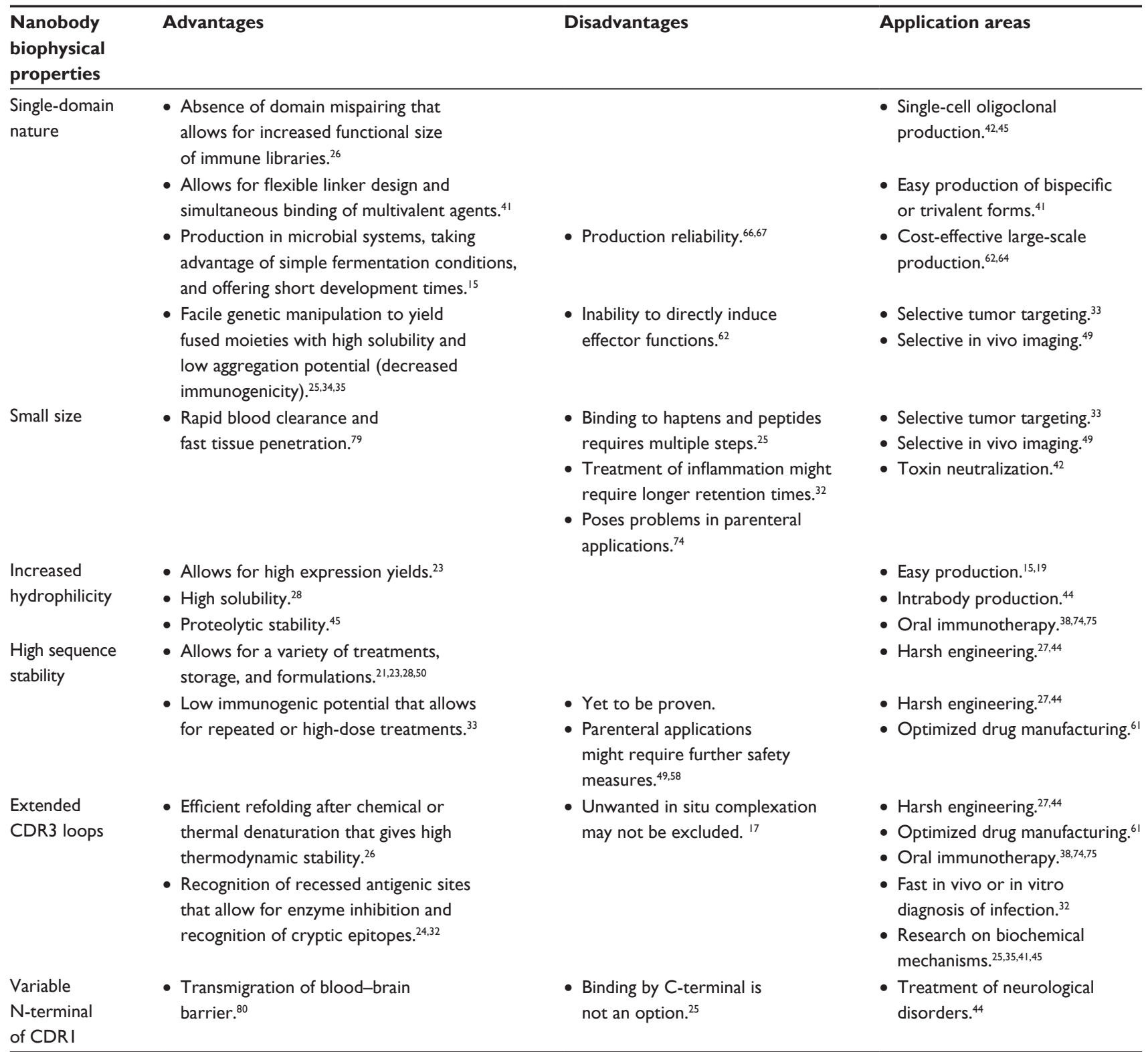

Note: Superscripted numbers refer to published papers that document each correlation.

Abbreviation: CDR, complementarity determining region.

The demonstrated, inferred, or possible $\mathrm{Nb}$ multimer constructs may be beneficial in serotherapeutics. Myeloma cell lines transfected with the antigen-specific $\mathrm{Nb}$ gene cloned in frame with the hinge $\mathrm{CH} 2$ and $\mathrm{CH} 3$ exons of human, and mouse or pig IgG, have been used to reconstruct chimeric HCAbs that specifically recognize the scorpion Androctonus australis hector toxin. ${ }^{76}$ The toxin neutralization capacity shown in mice exceeds the results provided from similar scFvbased constructs. In addition, several $\mathrm{Nbs}$ targeting bacterial toxins and snake venoms have been reported. ${ }^{42,77} \mathrm{~A}$ range of other human pathogens, such as Vibrio cholerae and Salmonella enterica, might also be suitable for $\mathrm{Nb}$ targeting.
$\mathrm{Nb}$ conjugates hold much promise for cancer immunotherapy and for the treatment of inflammatory diseases. Potential targets include cell surface proteins, cytokines, other secreted proteins, and even intracellular proteins. Chimeric reconstructs targeted against leukocyte ectoenzymes have been shown to completely block enzymatic activity and nicotinamide adenine dinucleotide (NAD)-induced cytotoxicity on the surface of T-cells, although at low inhibition rates; this may indicate slower tissue penetration capacity of the larger Fc-fusion protein. ${ }^{47}$ Nonetheless, the potential for autoimmune disease treatment has been clearly demonstrated. Lipopolysaccharide-Nbs efficiently block lipopolysaccharide 
binding and signaling to host cells, and they have been suggested for the treatment of sepsis. ${ }^{43}$ Conjugates to the epidermal growth factor receptor can block epidermal growth factor binding to its receptor, which can be used to treat solid tumors ${ }^{46}$ potency can be increased tenfold when using bivalent $\mathrm{Nb}$ constructs. Similar results have been reported for $\mathrm{Nbs}$ binding to tumor necrosis factor- $\alpha ;{ }^{41}$ the bivalent constructs exhibited 500 times more anti-tumor necrosis factor potency than the monovalent formats, and they even exceeded the potency of clinically used conventional Abs. These conjugates may be also utilized in imaging ${ }^{48,58}$ the total percent of injected activity per gram of tumor tissue is generally lower when compared to similar studies with conventional $\mathrm{Ab}$-constructs, but the generated specific contrast is at least ten times higher.

Like cytokines, other secreted molecules are accessible with $\mathrm{Nb}$ constructs, such as those developed against components of the blood-clotting cascade or the aggregation-prone proteins implicated in amyloidosis, where the employment of specific Nbs for inhibiting fibril formation seems to be a promising therapeutic strategy for Alzheimer's disease. ${ }^{55} \mathrm{Nbs}$ have been engineered to specifically recognize the activated form of the von Willebrand factor, a key component of the blood-clotting cascade that mediates the tethering of platelets to the vascular endothelium. ${ }^{78}$ Although the quantification of the target in serum samples was satisfactory, in vivo inhibition was limited. Another von Willebrand factor-specific $\mathrm{Nb}$ has recently completed a Phase I clinical trial as an antithrombotic agent, and it showed dose-dependent inhibition (www. Ablynx.com).

The $15 \mathrm{kDa}$ size of the $\mathrm{Nb}$ is well below the renal clearance limit of $60 \mathrm{kDa}$; for parenteral therapeutic applications, the serum half-lives should be increased. The use of bivalent formats allows for $\mathrm{Nb}$ ligation to large proteins such as albumin or Ig, which prolong in vivo retention to those of the ligature. ${ }^{41,46,74}$ Removal of Igs can have therapeutic benefits for patients with high titers of circulating auto-Abs (for example, in Goodpasture syndrome or in systemic lupus erythematosus). ${ }^{51}$ The chemical addition of polyethylene glycol (PEGylation) could also produce similar effects. The PEGylation of foot-and-mouth disease Nbs extended the serum half-life and enhanced in vitro neutralizing potency to levels above that of the hyperimmune serum; still, in vivo protection was not sufficient, suggesting that functionalization is required. ${ }^{45}$

The high sequence homology of Nbs to conventional VHs and their high conformational stability indicates a low immunogenic potential that was, thus far, demonstrated only in mice; ${ }^{33,41}$ however, conventional humanization remains indispensable. A radiolabeled anticarcinoembryonic antigen $\mathrm{Nb}$ was successfully humanized by loop grafting, demonstrating the potential of grafting specific antigen-binding loops to generate humanized $\mathrm{Nbs}$ that retain their targeting capacities for noninvasive in vivo tumor imaging. ${ }^{79}$ This approach offers a generic tool that allows for the complete standardization of both the production of clinical-grade Nbs and site-specific radiolabeling on the scaffold region. The main drawback of $\mathrm{Nbs}$ for imaging is their kidney retention, which limits the detection sensitivity in the vicinity of the kidneys (for example, in the pancreas), and induces a relatively high radiation dose to the kidney cells. ${ }^{7,49,58}$ In addition, parenteral applications may require further reduction in the immunogenic potential, possibly by using conventional Ablike Nbs, which have an even higher structural homology to the $\mathrm{VH}$ domains.

Nbs can surmount the blood-brain barrier. ${ }^{29}$ One of the Nbs selected from a llama, which was immunized with brain vascular endothelial cells, was found to undergo transcytosis and released on the basolateral side of the endothelial cells. ${ }^{29}$ In vivo studies showed that the $\mathrm{Nb}$ was efficiently transported across the blood-brain barrier and could even be used to carry any cargo into the brain, including the entire M13 phage particle..$^{80}$

The stability of Nbs is perfectly suited for the production of intrabodies that require their expression in the reducing environment of the cytoplasm. Bax-specific intrabodies have been developed to prevent the oxidative stress-induced apoptosis that is implicated in neurodegenerative diseases. ${ }^{44}$ The targeting of intracellular proteins may also proceed with the fusion of $\mathrm{Nbs}$ to peptides, thus mediating translocation across the cell membrane - although intrachain disulfide bonding could hamper unfolding and translocation. A transfectionbased expression strategy (although it is not very efficient) seems to be applicable for transgenic animals and plants, as well as in therapeutic settings where cells transfected in vitro are reapplied to the patient.

Extended CDR3 could potentially open the possibility of creating synthetic peptide mimics from Nbs. The X-ray structure of $\mathrm{Nbs}$ in a complex with lysozyme showed that the CDR3 fills the active site, mimicking a saccharide ligand..$^{25}$ Antilysozyme Nbs (peptibodies) have been shown to block lysozyme activity, although at higher concentrations than of the parental VHH. Still, the synthetic peptibodies may be delivered more efficiently to the cytosol than to the larger Nbs or the conventional $\mathrm{Ab}$ fragments. ${ }^{81}$ The binding of $\mathrm{Nbs}$ to small-sized antigens, such as haptens, may pose certain 
problems, as these require framework residues given that they bind in a groove or cavity at the VH-VL hydrophobic interface. ${ }^{25,45}$ Despite the absence of a VL, VHH form a deep cleft that involves residues from the CDRs; thus, binding could occur, though it depends more on electrostatic than hydrophobic effects. ${ }^{25} \mathrm{Nbs}$ raised against haptens using strong selection systems exhibit extremely high affinities that could be utilized in low-cost detection systems and in immunomodulation.

Nbs have been shown to bind to copper ions, ${ }^{25}$ raising new possibilities for the development of redox protein-based detection systems, such as solid-state electrochemical sensing, without the need for mediators. The ease of manipulation and the harsh engineering that Nbs can sustain make them perfect candidates as biorecognition elements in biosensor platforms. Although not within the scope of this review, it is worth noting that biosensors, using natural recognition processes for detecting targets with extremely low detection limits, have enjoyed low marketability since they have an operational versatility and stability that is largely dictated by the biological moiety that is immobilized on top of the physicochemical transducer. ${ }^{17} \mathrm{Nbs}$ can confer their extended shelf-life and ruggedness to biosensors, broadening their applicability to production control and environmental monitoring - two areas that (to date) only microorganisms can adequately serve at the expense of selectivity, sensitivity, and specificity. The ability of $\mathrm{Nbs}$ to recognize cryptic epitopes may open new avenues for environmental detection, especially in assessing pollution impact at the cellular or subcellular level, or even when elucidating the mechanisms of metabolic degradation and assimilation of endocrine disruptors, or of accumulating pesticides in sensitive ecosystems. The development of immunosensors using conventional $\mathrm{Ab}$ fragments has been proven to be challenging due to the low affinities expressed in the presence of binders, ${ }^{82}$ the option of producing multisensors using a series of high-avidity bispecific or trivalent $\mathrm{Nbs}$ that can be accommodated in the nanometer space may certainly enhance the simultaneous detection of many compounds with a single instrument. The high solubility and low aggregation potential of $\mathrm{Nbs}$ could reduce the use of reagents during measurements, while in situ regeneration of the sensor might be further facilitated for uses in long-term monitoring - although the use of optimized scaffolds or stress-driven selections (for example, temperature or chemical denaturing) might be necessary for assuring the optimal loading of the sensor. ${ }^{83}$

However, it should be noted that the small $\mathrm{Nb}$ size may not be suited for random coupling to solid surfaces unless advanced engineering produces formats that would permit directional immobilization. ${ }^{35}$ Notwithstanding, a prostatespecific antigen $\mathrm{Nb}$ was successfully immobilized to a surface plasmon resonance transducer by carbodiimide coupling to provide detection limits that were significantly lower than those of similar scFv platforms. ${ }^{35}$ The fusion protein retained its ability to self-assemble onto solid surface into a square lattice structure with the $\mathrm{Nb}$ pointing outwards from the protein lattice surface into the solution; this monomolecular protein lattice could be exploited as a sensing layer in various biosensor setups. In related research, different VHH constructs were immobilized onto commercial and custom-built sensor surfaces by metal chelation, biotin-streptavidin interactions, or covalent coupling. ${ }^{84}$ For the first time, the intrinsic stability was presented as an important biosensor design factor, showing experimentally that higher intrinsic stability offers higher resistance to harsh environments.

In the postgenomic era and with the high-throughput techniques available, the goal is to discriminate between all individual proteins from the proteome, including their splice variants and posttranslationally modified derivatives. Nbs may offer the means to attain this goal. More naturemimicking surfaces, such as lipid bilayers, may provide a more responsive host to $\mathrm{Nbs}$ and extend the range of applications in clinical diagnosis and environmental monitoring, where Nbs could be proven advantageous for maximizing protein loading to lower detection limits, reducing steric hindrance to lower noise levels, and optimizing dipolar and transmembrane potentials to increase sensitivity. ${ }^{17}$

\section{Conclusion}

Nature-derived Nbs represent a beneficial tool in Ab-based markets with ready-to-use potential. Numerous $\mathrm{Nbs}$ have already proven useful for basic and advanced research in therapy and diagnostics. In vitro and in vivo studies have shown, at least in principle, deep penetration into dense tissues, favorable kinetics for drug delivery, recognition of hidden epitopes, enhanced thermodynamic stability, and rapid elimination. The ability to tailor properties and pharmacokinetics with multivalent and fused constructs to suit the needs of the intended use places $\mathrm{Nbs}$ at the forefront of biomedical applications. Notwithstanding, a number of obstacles - including immunogenicity and functionalization - still have to be clinically proven and/or surmounted before clinical implementation becomes feasible.

\section{Acknowledgments}

The author thanks the anonymous reviewers for their insightful suggestions on improving the original manuscript. 


\section{Disclosure}

The author reports no conflicts of interest in this work.

\section{References}

1. Elbakri A, Nelson PN, Abu Odeh RO. The state of antibody therapy. Hum Immunol. 2010;71(12):1243-1250.

2. Beckman RA, Weiner LM, Davis HM. Antibody constructs in cancer therapy: protein engineering strategies to improve exposure in solid tumors. Cancer. 2007;109(2):170-179.

3. Natsume A, Niwa R, Satoh M. Improving effector functions of antibodies for cancer treatment: Enhancing ADCC and CDC. Drug Des Devel Ther. 2009;3:7-16.

4. Iyer U, Kadambi VJ. Antibody drug conjugates - Trojan horses in the war on cancer. J Pharmacol Toxicol Methods. 2011;64(3):207-212.

5. Hughes B. Antibody-drug conjugates for cancer: poised to deliver? Nat Rev Drug Discov. 2010;9(9):665-667.

6. Litvak-Greenfeld D, Benhar I. Risks and untoward toxicities of antibody-based immunoconjugates. Adv Drug Deliv Rev. 2012;64(15): 1782-1799.

7. Hildebrandt IJ, Gambhir SS. Molecular imaging applications for immunology. Clin Immunol. 2004;111(2):210-224.

8. Borrebaeck CA, Malmborg AC, Furebring C, et al. Kinetic analysis of recombinant antibody-antigen interactions: relation between structural domains and antigen binding. Biotechnology (NY). 1992;10(6): 697-698.

9. Lin S, Houston-Cummings NR, Prinz B, et al. A novel fragment of antigen binding (Fab) surface display platform using glycoengineered Pichia pastoris. J Immunol Methods. 2012;375(1-2):159-165.

10. Adams GP, Schier R, McCall AM, et al. High affinity restricts the localization and tumor penetration of single-chain fv antibody molecules. Cancer Res. 2001;61(12):4750-4755.

11. Nguyen A, Reyes AE, Zhang M, et al. The pharmacokinetics of an albumin-binding Fab (AB.Fab) can be modulated as a function of affinity for albumin. Protein Eng Des Sel. 2006;19(7):291-297.

12. Hamers-Casterman C, Atarhouch T, Muyldermans S, et al. Naturally occurring antibodies devoid of light chains. Nature. 1993;363(6428): 446-448.

13. Greenberg AS, Avila D, Hughes M, Hughes A, McKinney EC, Flajnik MF. A new antigen receptor gene family that undergoes rearrangement and extensive somatic diversification in sharks. Nature. 1995;374(6518):168-173.

14. Nuttall SD, Krishnan UV, Hattarki M, De Gori R, Irving RA, Hudson PJ. Isolation of the new antigen receptor from wobbegong sharks, and use as a scaffold for the display of protein loop libraries. Mol Immunol. 2001;38(4):313-326.

15. Muyldermans S. Nanobodies: natural single-domain antibodies. Annu Rev Biochem. 2013;82:775-797.

16. Mogoutov A, Kahane B. Data search strategy for science and technology emergence: a scalable and evolutionary query for nanotechnology tracking. Res Policy. 2007;36:893-903.

17. Siontorou CG, Batzias FA. Innovation in biotechnology: moving from academic research to product development - the case of biosensors. Crit Rev Biotechnol. 2010;30(2):79-98.

18. Leydesdorff L, Rafols I. A global map of science based on the ISI subject categories. J Am Soc Inf Sci Technol. 2009;60:348-362.

19. Muyldermans S, Atarhouch T, Saldanha J, Barbosa JA, Hamers R. Sequence and structure of $\mathrm{VH}$ domain from naturally occurring camel heavy chain immunoglobulins lacking light chains. Protein Eng. 1994;7(9):1129-1135.

20. Desmyter A, Transue TR, Ghahroudi MA, et al. Crystal structure of a camel single-domain $\mathrm{VH}$ antibody fragment in complex with lysozyme. Nat Struct Biol. 1996;3(9):803-811.

21. van der Linden RH, Frenken LG, de Geus B, et al. Comparison of physical chemical properties of llama VHH antibody fragments and mouse monoclonal antibodies. Biochim Biophys Acta. 1999;1431(1):37-46.
22. Vu KB, Ghahroudi MA, Wyns L, Muyldermans S. Comparison of llama VH sequences from conventional and heavy chain antibodies. Mol Immunol. 1997;34(16-17):1121-1131.

23. Arbabi Ghahroudi M, Desmyter A, Wyns L, Hamers R, Muyldermans S. Selection and identification of single domain antibody fragments from camel heavy-chain antibodies. FEBS Lett. 1997;414(3):521-526.

24. Lauwereys M, Arbabi Ghahroudi M, Desmyter A, et al. Potent enzyme inhibitors derived from dromedary heavy-chain antibodies. EMBO J. 1998;17(13):3512-3520.

25. Spinelli S, Frenken LG, Hermans P, et al. Camelid heavy-chain variable domains provide efficient combining sites to haptens. Biochemistry. 2000;39(6):1217-1222.

26. Frenken LG, van der Linden RH, Hermans PW, et al. Isolation of antigen specific llama VHH antibody fragments and their high level secretion by Saccharomyces cerevisiae. J Biotechnol. 2000;78(1):11-21.

27. Pérez JM, Renisio JG, Prompers JJ, et al. Thermal unfolding of a llama antibody fragment: a two-state reversible process. Biochemistry. 2001;40(1):74-83.

28. Dumoulin M, Conrath K, Van Meirhaeghe A, et al. Single-domain antibody fragments with high conformational stability. Protein Sci. 2002;11(3):500-515.

29. Muruganandam A, Tanha J, Narang S, Stanimirovic D. Selection of phage-displayed llama single-domain antibodies that transmigrate across human blood-brain barrier endothelium. FASEB J. 2002;16(2): $240-242$.

30. Verheesen P, ten Haaft MR, Lindner N, Verrips CT, de Haard JJ. Beneficial properties of single-domain antibody fragments for application in immunoaffinity purification and immuno-perfusion chromatography. Biochim Biophys Acta. 2003;1624(1-3):21-28.

31. Stanfield RL, Dooley H, Flajnik MF, Wilson IA. Crystal structure of a shark single-domain antibody $\mathrm{V}$ region in complex with lysozyme. Science. 2004;305(5691):1770-1773.

32. Stijlemans B, Conrath K, Cortez-Retamozo V, et al. Efficient targeting of conserved cryptic epitopes of infectious agents by single domain antibodies. African trypanosomes as paradigm. $J$ Biol Chem. 2004;279(2):1256-1261.

33. Cortez-Retamozo V, Backmann N, Senter PD, et al. Efficient cancer therapy with a nanobody-based conjugate. Cancer Res. 2004;64(8): 2853-2857.

34. Zhang J, Tanha J, Hirama T, et al. Pentamerization of single-domain antibodies from phage libraries: a novel strategy for the rapid generation of high-avidity antibody reagents. $J$ Mol Biol. 2004;335(1):49-56.

35. Pleschberger M, Saerens D, Weigert S, et al. An S-layer heavy chain camel antibody fusion protein for generation of a nanopatterned sensing layer to detect the prostate-specific antigen by surface plasmon resonance technology. Bioconjug Chem. 2004;15(3):664-671.

36. Joosten V, Roelofs MS, van den Dries N, et al. Production of bifunctional proteins by Aspergillus awamori: llama variable heavy chain antibody fragment $(\mathrm{V}(\mathrm{HH})) \mathrm{R} 9$ coupled to Arthromyces ramosus peroxidase (ARP). J Biotechnol. 2005;120(4):347-359.

37. Dolk E, van der Vaart M, Lutje Hulsik D, et al. Isolation of llama antibody fragments for prevention of dandruff by phage display in shampoo. Appl Environ Microbiol. 2005;71(1):442-450.

38. van der Vaart JM, Pant N, Wolvers D, et al. Reduction in morbidity of rotavirus induced diarrhoea in mice by yeast produced monovalent llama-derived antibody fragments. Vaccine. 2006;24(19): $4130-4137$.

39. Rothbauer U, Zolghadr K, Tillib S, et al. Targeting and tracing antigens in live cells with fluorescent nanobodies. Nat Methods. 2006;3(11): $887-889$.

40. Rahbarizadeh F, Rasaee MJ, Forouzandeh M, Allameh AA. Over expression of anti-MUC1 single-domain antibody fragments in the yeast Pichia pastoris. Mol Immunol. 2006;43(5):426-435.

41. Coppieters K, Dreier T, Silence K, et al. Formatted anti-tumor necrosis factor alpha $\mathrm{VHH}$ proteins derived from camelids show superior potency and targeting to inflamed joints in a murine model of collagen-induced arthritis. Arthritis Rheum. 2006;54(6):1856-1866. 
42. Harrison RA, Hasson SS, Harmsen M, Laing GD, Conrath K, Theakston RD. Neutralisation of venom-induced haemorrhage by $\mathrm{IgG}$ from camels and llamas immunised with viper venom and also by endogenous, non-IgG components in camelid sera. Toxicon. 2006;47(3):364-368.

43. El Khattabi M, Adams H, Heezius E, et al. Llama single-chain antibody that blocks lipopolysaccharide binding and signaling: prospects for therapeutic applications. Clin Vaccine Immunol. 2006;13(10):1079-1086.

44. Gueorguieva D, Li S, Walsh N, Mukerji A, Tanha J, Pandey S. Identification of single-domain, Bax-specific intrabodies that confer resistance to mammalian cells against oxidative-stress-induced apoptosis. FASEB J. 2006;20(14):2636-2638.

45. Harmsen MM, van Solt CB, Fijten HP, et al. Passive immunization of guinea pigs with llama single-domain antibody fragments against foot-and-mouth disease. Vet Microbiol. 2007;120(3-4):193-206.

46. Roovers RC, Laeremans T, Huang L, et al. Efficient inhibition of EGFR signaling and of tumour growth by antagonistic anti-EFGR Nanobodies. Cancer Immunol Immunother. 2007;56(3):303-317.

47. Koch-Nolte F, Reyelt J, Schössow B, et al. Single domain antibodies from llama effectively and specifically block T cell ecto-ADP-ribosyltransferase ART2.2 in vivo. FASEB J. 2007;21(13):3490-3498.

48. Achour I, Cavelier P, Tichit M, Bouchier C, Lafaye P, Rougeon F. Tetrameric and homodimeric camelid IgGs originate from the same IgH locus. J Immunol. 2008;181(3):2001-2009.

49. Huang L, Gainkam LO, Caveliers V, et al. SPECT imaging with 99mTc-labeled EGFR-specific nanobody for in vivo monitoring of EGFR expression. Mol Imaging Biol. 2008;10(3):167-175.

50. Vuchelen A, O’Day E, De Genst E, et al. (1)H, (13)C and (15)N assignments of a camelid nanobody directed against human alpha-synuclein. Biomol NMR Assign. 2009;3(2):231-233.

51. Klooster R, Eman MR, le Duc Q, et al. Selection and characterization of KDEL-specific VHH antibody fragments and their application in the study of ER resident protein expression. J Immunol Methods. 2009;342(1-2):1-12.

52. Van den Abbeele A, De Clercq S, De Ganck A, et al. A llama-derived gelsolin single-domain antibody blocks gelsolin-G-actin interaction. Cell Mol Life Sci. 2010;67(9):1519-1535.

53. Flajnik MF, Kasahara M. Origin and evolution of the adaptive immune system: genetic events and selective pressures. Nat Rev Genet. 2010;11(1):47-59.

54. Kirchhofer A, Helma J, Schmidthals K, et al. Modulation of protein properties in living cells using nanobodies. Nat Struct Mol Biol. 2010;17(1):133-138.

55. Domanska K, Vanderhaegen S, Srinivasan V, et al. Atomic structure of a nanobody-trapped domain-swapped dimer of an amyloidogenic beta2-microglobulin variant. Proc Natl Acad Sci U S A. 2011;108(4): 1314-1319.

56. Rasmussen SG, Choi HJ, Fung JJ, et al. Structure of a nanobodystabilized active state of the $\beta(2)$ adrenoceptor. Nature. 2011;469(7329): 175-180.

57. Caussinus E, Kanca O, Affolter M. Fluorescent fusion protein knockout mediated by anti-GFP nanobody. Nat Struct Mol Biol. 2012;19(1): $117-121$.

58. Broisat A, Hernot $\mathrm{S}$, Toczek J, et al. Nanobodies targeting mouse/human VCAM1 for the nuclear imaging of atherosclerotic lesions. Circ Res. 2012;110(7):927-937.

59. Padlan EA. Anatomy of the antibody molecule. Mol Immunol. 1994;31(3):169-217.

60. Reiter Y, Schuck P, Boyd LF, Plaksin D. An antibody single-domain phage display library of a native heavy chain variable region: isolation of functional single-domain $\mathrm{VH}$ molecules with a unique interface. J Mol Biol. 1999;290(3):685-698.

61. Holt LJ, Herring C, Jespers LS, Woolven BP, Tomlinson IM. Domain antibodies: proteins for therapy. Trends Biotechnol. 2003;21(11): 484-490.

62. Nguyen VK, Desmyter A, Muyldermans S. Functional heavy-chain antibodies in Camelidae. Adv Immunol. 2001;79:261-296.
63. Govaert J, Pellis M, Deschacht N, et al. Dual beneficial effect of interloop disulfide bond for single domain antibody fragments. J Biol Chem. 2012;287(3):1970-1979.

64. Saerens D, Stijlemans B, Baral TN, et al. Parallel selection of multiple anti-infectome Nanobodies without access to purified antigens. J Immunol Methods. 2008;329(1-2):138-150.

65. Abbady AQ, Al-Mariri A, Zarkawi M, Al-Assad A, Muyldermans S. Evaluation of a nanobody phage display library constructed from a Brucellaimmunised camel. Vet Immunol Immunopathol. 2011;142(1-2):49-56.

66. Goldman ER, Anderson GP, Liu JL, et al. Facile generation of heatstable antiviral and antitoxin single domain antibodies from a semisynthetic llama library. Anal Chem. 2006;78(24):8245-8255.

67. Monegal A, Ami D, Martinelli C, et al. Immunological applications of single-domain llama recombinant antibodies isolated from a naïve library. Protein Eng Des Sel. 2009;22(4):273-280.

68. Gerngross TU. Advances in the production of human therapeutic proteins in yeasts and filamentous fungi. Nat Biotechnol. 2004;22(11): 1409-1414.

69. Thomassen YE, Meijer W, Sierkstra L, Verrips CT. Large-scale production of VHH antibody fragments by Saccharomyces cerevisiae. Enzyme Microb Technol. 2002;3(3):273-278.

70. Sethuraman N, Stadheim TA. Challenges in therapeutic glycoprotein production. Curr Opin Biotechnol. 2006;17(4):341-346.

71. Ryckaert S, Pardon E, Steyaert J, Callewaert N. Isolation of antigenbinding camelid heavy chain antibody fragments (nanobodies) from an immune library displayed on the surface of Pichia pastoris. J Biotechnol. 2010;145(2):93-98.

72. Hagihara Y, Mine S, Uegaki K. Stabilization of an immunoglobulin fold domain by an engineered disulfide bond at the buried hydrophobic region. J Biol Chem. 2007;282(50):36489-36495.

73. Saerens D, Conrath K, Govaert J, Muyldermans S. Disulfide bond introduction for general stabilization of immunoglobulin heavy-chain variable domains. J Mol Biol. 2008;377(2):478-488.

74. Harmsen MM, van Solt CB, van Zijderveld-van Bemmel AM, Niewold TA, van Zijderveld FG. Selection and optimization of proteolytically stable llama single-domain antibody fragments for oral immunotherapy. Appl Microbiol Biotechnol. 2006;72(3):544-551.

75. Pant N, Hultberg A, Zhao Y, et al. Lactobacilli expressing variable domain of llama heavy-chain antibody fragments (lactobodies) confer protection against rotavirus-induced diarrhea. J Infect Dis. 2006;194(11):1580-1588.

76. Hmila I, Abdallah RBA, Saerens D, et al. VHH, bivalent domains and chimeric Heavy chain-only antibodies with high neutralizing efficacy for scorpion toxin AahI'. Mol Immunol. 2008;45(14):3847-3856

77. Hussack G, Arbabi-Ghahroudi M, van Faassen H, et al. Neutralization of Clostridium difficile toxin A with single-domain antibodies targeting the cell receptor binding domain. J Biol Chem. 2011;286(11): 8961-8976

78. Hulstein JJ, de Groot PG, Silence K, Veyradier A, Fijnheer R, Lenting PJ. A novel nanobody that detects the gain-of-function phenotype of von Willebrand factor in ADAMTS13 deficiency and von Willebrand disease type 2B. Blood. 2005;106(9):3035-3042.

79. Vaneycken I, Govaert J, Vincke C, et al. In vitro analysis and in vivo tumor targeting of a humanized, grafted nanobody in mice using pinhole SPECT/micro-CT. J Nucl Med. 2010;51(7):1099-1106.

80. Abulrob A, Sprong H, Van Bergen en Henegouwen P, Stanimirovic D. The blood-brain barrier transmigrating single domain antibody: mechanisms of transport and antigenic epitopes in human brain endothelial cells. J Neurochem. 2005;95(4):1201-1214.

81. Marquardt A, Muyldermans S, Przybylski M. A synthetic camel antilysozyme peptide antibody (peptibody) with flexible loop structure identified by high-resolution affinity mass spectrometry. Chemistry. 2006;12(7):1915-1923.

82. Backmann N, Zahnd C, Huber F, et al. A label-free immunosensor array using single-chain antibody fragments. Proc Natl Acad Sci U SA. 2005;102(41):14587-14592. 
83. Steinhauer C, Wingren C, Hager AC, Borrebaeck CA. Single framework recombinant antibody fragments designed for protein chip applications. BioTechniques. 2002;Suppl:38-45.
84. Saerens D, Frederix F, Reekmans G, et al. Engineering camel singledomain antibodies and immobilization chemistry for human prostatespecific antigen sensing. Anal Chem. 2005;77(23):7547-7555.

\section{Publish your work in this journal}

The International Journal of Nanomedicine is an international, peerreviewed journal focusing on the application of nanotechnology in diagnostics, therapeutics, and drug delivery systems throughou the biomedical field. This journal is indexed on PubMed Central, MedLine, CAS, SciSearch $\AA$, Current Contents ${ } /$ Clinical Medicine,
Journal Citation Reports/Science Edition, EMBase, Scopus and the Elsevier Bibliographic databases. The manuscript management system is completely online and includes a very quick and fair peer-review system, which is all easy to use. Visit http://www.dovepress.com/ testimonials.php to read real quotes from published authors.

Submit your manuscript here: http://www.dovepress.com/international-journal-of-nanomedicine-journal 